\title{
Unique continuation for differential equations of Schrödinger's type
}

\author{
R. RegBaoui
}

We prove that the strong unique continuation property holds for the differential inequality $|\Delta u(x)| \leq V(x)|\nabla u(x)|$, where $V$ is a function in $L_{l o c}^{r}(\Omega)$ with $r>\frac{7 n-2}{6}, n \geq 3$, and $\Omega$ a connected open subset of $\mathbb{R}^{n}$. If $n>5$ our result improves that of Wolff [6] who got $r \geq \operatorname{Max}\left(n, \frac{3 n-4}{2}\right)$.

\section{Introduction and statement of results.}

Let $\Omega$ be a connected open subset of $\mathbb{R}^{n}$, and $V$ a positive function on $\Omega$. We say that the differential inequality

$$
|\Delta u(x)| \leq V(x)|\nabla u(x)|
$$

has the strong unique continuation property (s.u.c.p) if every solution of (1.1) that vanishes to infinite order at a point of $\Omega$ must be identically zero. We recall that a function $u \in L_{l o c}^{2}(\Omega)$ is said to vanish to infinite order at a point $x_{0} \in \Omega$ if it satisfies

$$
\int_{\left|x-x_{0}\right|<R}|u(x)|^{2} d x=\mathcal{O}\left(R^{N}\right) \text { for all } N>0 \text { as } R \rightarrow 0 .
$$

There is an extensive literature on unique continuation for differential inequalities like (1.1). We refer the reader to ([1], [2], [3], [4], [7]) for more details on the subject.

In his paper [6], Wolff proved that (1.1) has the (s.u.c.p) if $V \in L_{l o c}^{r}(\Omega)$ with $r \geq \operatorname{Max}\left(n, \frac{3 n-4}{2}\right)$. We note that this result is optimal when $n \leq 4$ since as it can be shown by elementary examples, the (s.u.c.p) fails for $r<n$. Counterexamples due to Wolff [8] show that the (s.u.c.p) fails also for $r=n, n>4$. A natural question is then: the exponent $r=n+\varepsilon$ is it the optimal one ?. Although we are unable to answer this question, we prove the following: 
Theorem 1.1. Let $r>\frac{7 n-2}{6}, n \geq 3$, and let $u \in W_{\text {loc }}^{2,2}(\Omega)$ be a solution of (1.1) with $V \in L_{\text {loc }}^{r}(\Omega)$. Suppose that $u$ vanishes to infinite order at a point $x_{0} \in \Omega$, i.e., satisfies (1.2). Then u vanishes identically in $\Omega$.

When $n>5$ theorem 1.1 is an improvement of Wolff's result since $\frac{7 n-2}{6}<$ $\operatorname{Max}\left(n, \frac{3 n-4}{2}\right)$ if $n>5$. It's not clear to us wether the method we have employed here allows one to prove the (s.u.c.p) for $V \in L_{l o c}^{r}$ with $r<$ $\frac{7 n-2}{6}, n \geq 5$ (see remark 1.4).

Before proving theorem 1.1, we show that if $u$ is as in theorem 1.1, then $u$ has faster than "exponential vanishing" at $x_{0}$, namely:

Theorem 1.2. Let $r>\frac{7 n-2}{6}, n \geq 3$, and let $u \in W_{\text {loc }}^{2,2}(\Omega)$ be a solution of (1.1) with $V \in L_{\text {loc }}^{r}(\Omega)$. Suppose that $u$ vanishes to infinite order at a point $x_{0} \in \Omega$, i.e, satisfies (1.2). Then u satisfes, for all $|\alpha| \leq 1$,

$$
\int_{\left|x-x_{0}\right|<R}\left|D^{\alpha} u(x)\right|^{2} d x=\mathcal{O}\left(e^{-N R^{-1}}\right) \text { for all } N>0 \text { as } R \rightarrow 0 .
$$

Theorem 1.2 allows us to conclude theorem 1.1 from the following:

Theorem 1.3. Let $p=\frac{2 n}{n+2}, n \geq 3$, and let $u \in W_{\text {loc }}^{2, p}(\Omega)$ be a solution of (1.1) with $V \in L_{\text {loc }}^{n}(\Omega)$. Suppose that $u$ satisfies, for some $x_{0} \in \Omega$ and all $|\alpha| \leq 1$,

$$
\int_{\left|x-x_{0}\right|<R}\left|D^{\alpha} u(x)\right|^{2} d x=\mathcal{O}\left(e^{-N R^{-1}}\right) \text { for all } N>0 \text { as } R \rightarrow 0 .
$$

Then $u$ vanishes identically in $\Omega$.

Remark 1.4. a) For technical reasons we have supposed $u \in W_{l o c}^{2,2}$ in theorem 1.1. By using interpolation inequalities in the proof of theorem 1.2 (to obtain (2.33) ), one can see that theorem 1.1 remains valid if we suppose $u \in W_{l o c}^{2, m}$ with $\frac{1}{m}=\frac{1}{2}+\frac{1}{r}, r>\frac{7 n-2}{6}$.

b) The reason for we make the restriction $r>\frac{7 n-2}{6}$ in theorem 1.1, is that the exponent $\frac{7 n-2}{6}$ is the smallest one allowing us to obtain exponential decay of the form (1.3) since such a decay is needed to apply theorem 1.3. The exponent $r=n+\varepsilon$ gives only a decay of the form

$$
\int_{\left|x-x_{0}\right|<R}\left|D^{\alpha} u(x)\right|^{2} d x=\mathcal{O}\left(e^{-R^{-a}}\right) \text { as } R \rightarrow 0
$$

for some positive $a($ not necessary $\geq 1)$. 
Unique continuation for differential equations of Schrödinger's type 305

To prove theorem 1.3 we use a modified Carleman method due to Wolff [7], where the main tool is a lemma concerning concentration of measures in $\mathbb{R}^{n}$.

\section{Proof of the results.}

As mentioned above, the principal point in the proof of theorem 1.3 is the following lemma due to Wolff [7]:

Lemma 2.1 (Wolff [7], lemma 1). Let $\mu$ be a positive measure in $\mathbb{R}^{n}$ with faster than exponential decay, i.e.,

$$
\lim _{T \rightarrow \infty} T^{-1} \log \mu(\{x:|x| \geq T\})=-\infty .
$$

Define $\mu_{k}$ by $d \mu_{k}(x)=e^{k \cdot x} d \mu(x)$. Suppose $\mathcal{B}$ is a convex body in $\mathbb{R}^{n}$. Then there is a sequence $\left\{k_{j}\right\} \subset \mathcal{B}$ and, for each $j$, a convex body $E_{k_{j}}$ with

$$
\mu_{k_{j}}\left(\mathbb{R}^{n} \backslash E_{k_{j}}\right) \leq \frac{1}{2}\left\|\mu_{k_{j}}\right\|
$$

such that $\left\{E_{k_{j}}\right\}$ are pairwise disjoint and satisfying

$$
\sum_{j}\left|E_{k_{j}}\right|^{-1} \geq C|\mathcal{B}|
$$

where $C$ is a positive constant depending only on $n$, and where $|\mathcal{B}|,\left|E_{k_{j}}\right|$ denote the Lebesgue measures of $\mathcal{B}$ and $E_{k_{j}}$.

We need also the following Carleman-type estimate which is proved in Wolff [7].

Lemma 2.2 (Wolff [7], Lemma 6.2). Let $p=\frac{2 n}{n+2}, n \geq 3$. If $k \in$ $\mathbb{R}^{n}, k \neq 0, E \subset \mathbb{R}^{n}$ with $|E| \geq|k|^{-n}$, then for all $u \in W^{2, p}\left(\mathbb{R}^{n}\right)$ with compact support, and for all $\theta>\frac{n-2}{n(n-1)}$,

$$
\left\|e^{k \cdot x} \nabla u\right\|_{L^{2}(E)} \leq C_{\theta}\left(|k|^{n}|E|\right)^{\theta}\left\|e^{k \cdot x} \Delta u\right\|_{L^{p}}
$$

where $C_{\theta}$ is a positive constant depending only on $n$ and $\theta$.

Proof of theorem 1.3. Let $u$ as in theorem 1.3. We may suppose that $x_{0}=0$, and using a weak unique continuation theorem of Wolff [7](Theorem 1), it suffices to prove that $\nabla u \equiv 0$ in a neighborhood of 0 . 
Let $R_{0}>0$ sufficiently large such that $B\left(0, R_{0}^{-1}\right) \subset \Omega$. For $|x|>R_{0}$ define $U$ by $U(x)=u\left(\frac{x}{|x|^{2}}\right)$. Then it suffices to prove that $\nabla U \equiv 0$ for large $|x|$. But since the problem is rotation-invariant it will suffice to prove that $\nabla U \equiv 0$ in the cone:

$$
\Gamma=\left\{x:|x|>20 R_{0}\right\} \cap\left\{x: x_{n} \geq 2 \sqrt{x_{1}^{2}+\cdots+x_{n-1}^{2}}\right\}
$$

$\left(R_{0}\right.$ large enough $)$.

An easy computation shows that $U \in W_{l o c}^{2, p}\left(\mathbb{R}^{n} \backslash B\left(0, R_{0}\right)\right)$ and satisfies

$$
|\Delta U(x)| \leq\left(W(x)+2(n-1)|x|^{-1}\right)|\nabla U(x)|
$$

where $W \in L^{n}\left(\mathbb{R}^{n} \backslash B\left(0, R_{0}\right)\right)$.

Since $u$ satifies (1.3), we get without difficulty, for $|\alpha| \leq 1$,

$$
\int_{|x|>R}\left|D^{\alpha} U(x)\right|^{2} d x=\mathcal{O}\left(e^{-N R}\right) \text { for all } N>0 \text { as } R \rightarrow \infty \text {. }
$$

It follows from Hölder's inequality and the fact that $W \in L^{n}\left(\mathbb{R}^{n} \backslash B\left(0, R_{0}\right)\right)$ that

$$
\int_{|x|>R}(W(x)|\nabla U(x)|)^{p} d x=\mathcal{O}\left(e^{-N R}\right) \text { for all } N>0 \text { as } R \rightarrow \infty .
$$

By using the following inequality

$$
\left(\int_{R<|x|<2 R}\left(|x|^{-1}|\nabla U(x)|\right)^{p} d x\right)^{1 / p} \leq\left(\int_{R<|x|<2 R}|\nabla U(x)|^{2} d x\right)^{1 / 2}
$$

and a dyadic decomposition of the set $\{x:|x|>R\}$, we get

$$
\int_{|x|>R}\left(|x|^{-1}|\nabla U(x)|\right)^{p} d x=\mathcal{O}\left(e^{-N R}\right) \text { for all } N>0 \text { as } R \rightarrow \infty .
$$

It follows then

$$
\int_{|x|>R}\left(\left(W(x)+2(n-1)|x|^{-1}\right)|\nabla U(x)|\right)^{p} d x=\mathcal{O}\left(e^{-N R}\right)
$$

for all $N>0$ as $R \rightarrow \infty$.

The estimate (2.4) in lemma 2.2 was stated for functions in $W^{2, p}$ with compact supports, but a standard limiting argument using (2.6), (2.7) and 
the inequality $|\Delta U(x)| \leq\left(W(x)+2(n-1)|x|^{-1}\right)|\nabla U(x)|$ shows that it is also true for the function $\phi U$, where $\phi \in C_{0}^{\infty}\left(\mathbb{R}^{n}\right)$ such that $\phi(x)=0$ if $|x|<R_{0}$, and $\phi(x)=1$ if $|x|>2 R_{0}$. Then

$$
\left\|e^{k \cdot x} \nabla(\phi U)\right\|_{L^{2}(E)} \leq C_{\theta}\left(|k|^{n}|E|\right)^{\theta}\left\|e^{k \cdot x} \Delta(\phi U)\right\|_{L^{p}} .
$$

Let $M>0$ sufficently large to be chosen later, and let

$$
\mathcal{B}=B\left(M e_{n}, \frac{M}{10}\right)
$$

where $e_{n}$ is the unit vector $(0, \cdots, 1) \in \mathbb{R}^{n}$. Thus $\mathcal{B}$ is a convex body of $\mathbb{R}^{n}$ with $|\mathcal{B}|=C M^{n}$, where $C$ is a positive constant depending only on $n$.

By Leibniz formula we have $\Delta(\phi U)=\phi \Delta U+U \Delta \phi+2 \nabla U \cdot \nabla \phi$. Hence by using (2.5), we get from (2.8), for all $k \in \mathbb{R}^{n}$ with $p k \in \mathcal{B}$,

$$
\begin{aligned}
& \left\|e^{k \cdot x} \nabla(\phi U)\right\|_{L^{2}(E)} \leq \\
& \quad C_{\theta}\left(M^{n}|E|\right)^{\theta}\left(\left\|e^{k \cdot x}\left(W(x)+2(n-1)|x|^{-1}\right)|\nabla(\phi U)|\right\|_{L^{p}}+\mathcal{R}\right)
\end{aligned}
$$

where

$$
\begin{aligned}
\mathcal{R}=\left\|e^{k \cdot x} U \Delta \phi\right\|_{L^{p}}+2 \| e^{k \cdot x} \nabla \phi & \cdot \nabla U \|_{L^{p}}+ \\
& +\left\|e^{k \cdot x}\left(W(x)+2(n-1)|x|^{-1}\right) U \nabla \phi\right\|_{L^{p}},
\end{aligned}
$$

and $C_{\theta}$ is a new positive constant depending only on $n$ and $\theta$.

We suppose that $\nabla U \not \equiv 0$ in $\Gamma$, and we will prove that this leads to a contradiction.

We claim that

$$
\mathcal{R} \leq\left\|e^{k \cdot x}\left(W(x)+2(n-1)|x|^{-1}\right)|\nabla(\phi U)|\right\|_{L^{p}} .
$$

Indeed, we have

$$
\begin{aligned}
& \left\|e^{k \cdot x}\left(W(x)+2(n-1)|x|^{-1}\right)|\nabla(\phi U)|\right\|_{L^{p}}^{p} \geq \\
& \qquad \int_{x \in \Gamma} e^{p k \cdot x}\left(\left(W(x)+2(n-1)|x|^{-1}\right)|\nabla U|\right)^{p} d x,
\end{aligned}
$$

but for $x \in \Gamma$ and $p k \in \mathcal{B}$ we have $p k \cdot x \geq 6 M R_{0}$. Hence

$$
\begin{aligned}
& \left\|e^{k \cdot x}\left(W(x)+2(n-1)|x|^{-1}\right)|\nabla(\phi U)|\right\|_{L^{p}}^{p} \geq \\
& e^{6 M R_{0}} \int_{x \in \Gamma}\left(\left(W(x)+2(n-1)|x|^{-1}\right)|\nabla U|\right)^{p} d x .
\end{aligned}
$$


On the other hand, for $p k \in \mathcal{B}$, we have

$$
\mathcal{R} \leq C R_{0}^{-1} e^{4 M R_{0} / p}\left(\|U\|_{W^{1, p}}+\|W U\|_{L^{p}}\right),
$$

where $\|U\|_{W^{1, p}}$ is the $W^{1, p}$-norm of $U$ in the set $\left\{x: R_{0}<|x|<2 R_{0}\right\}$, and $\|W U\|_{L^{p}}$ is the $L^{p}$-norm of $W U$ in the same set. $C$ is a positive constant depending only on $n$.

It follows that

$$
\begin{aligned}
& \frac{\mathcal{R}}{\left\|e^{k \cdot x}\left(W(x)+2(n-1)|x|^{-1}\right)|\nabla(\phi U)|\right\|_{L^{p}}} \leq \\
& \frac{C R_{0}^{-1} e^{-2 M R_{0} / p}\left(\|U\|_{W^{1, p}}+\|W U\|_{L^{p}}\right)}{\left(\int_{x \in \Gamma}\left(\left(W(x)+2(n-1)|x|^{-1}\right)|\nabla U|\right)^{p} d x\right)^{1 / p}}
\end{aligned}
$$

$R_{0}$ being fixed, if $M$ is large enough we obtain

$$
\frac{\mathcal{R}}{\left\|e^{k \cdot x}\left(W(x)+2(n-1)|x|^{-1}\right)|\nabla(\phi U)|\right\|_{L^{p}}} \leq 1
$$

that's

$$
\mathcal{R} \leq\left\|e^{k \cdot x}\left(W(x)+2(n-1)|x|^{-1}\right)|\nabla(\phi U)|\right\|_{L^{p}}
$$

as claimed.

Then (2.9) becomes

(2.10) $\left\|e^{k \cdot x} \nabla(\phi U)\right\|_{L^{2}(E)} \leq$

$$
2 C_{\theta}\left(M^{n}|E|\right)^{\theta}\left\|e^{k \cdot x}\left(W(x)+2(n-1)|x|^{-1}\right)|\nabla(\phi U)|\right\|_{L^{p}} .
$$

Define a positive measure $\mu$ by

$$
d \mu(x)=\left(\left(W(x)+2(n-1)|x|^{-1}\right)|\nabla(\phi U)|\right)^{p} d x .
$$

It follows from (2.7) that $\mu$ has faster than exponential decay. Hence by lemma 2.1 there is a sequence $\left\{k_{j}\right\}$ with $\left\{p k_{j}\right\} \subset \mathcal{B}$, for each $j$ a convex body $E_{k_{j}}$ such that $E_{k_{j}}$ are pairwise disjoint and satisfying:

$$
\begin{gathered}
\left\|e^{k_{j} \cdot x}\left(W(x)+2(n-1)|x|^{-1}\right)|\nabla(\phi U)|\right\|_{L^{p}}^{p} \leq \\
2\left\|e^{k_{j} \cdot x}\left(W(x)+2(n-1)|x|^{-1}\right)|\nabla(\phi U)|\right\|_{L^{p}\left(E_{k_{j}}\right)}^{p}
\end{gathered}
$$

and

$$
\sum_{j}\left(M^{n}\left|E_{k_{j}}\right|\right)^{-1} \geq C
$$


Unique continuation for differential equations of Schrödinger's type 309

where $C$ is a positive constant depending only on $n$.

We may suppose $\left|E_{k_{j}}\right| \geq M^{-n}$ (else drop all of them but one and enlarge it to have volume $=M^{-n}$ ).

If we take $E=E_{k_{j}}$ in (2.10) and combine with (2.11) we get

$$
\begin{aligned}
& \left\|e^{k_{j} \cdot x} \nabla(\phi U)\right\|_{L^{2}\left(E_{k_{j}}\right)} \leq \\
& \quad 4 C_{\theta}\left(M^{n}\left|E_{k_{j}}\right|\right)^{\theta}\left\|e^{k_{j} \cdot x}\left(W(x)+2(n-1)|x|^{-1}\right)|\nabla(\phi U)|\right\|_{L^{p}\left(E_{k_{j}}\right)} .
\end{aligned}
$$

But by Hölder's inequality we have, for all $\varepsilon>0$,

$$
\begin{aligned}
& \left\|e^{k_{j} \cdot x}\left(W(x)+2(n-1)|x|^{-1}\right)|\nabla(\phi U)|\right\|_{L^{p}\left(E_{k_{j}}\right)} \leq \\
& \quad\left(\|W\|_{L^{n}\left(Y_{j}\right)}+2(n-1)\left|E_{k_{j}}\right|^{\frac{\varepsilon}{n(n+\varepsilon)}}\left\||x|^{-1}\right\|_{L^{n+\varepsilon}\left(Y_{j}\right)}\right)\left\|e^{k_{j} \cdot x} \nabla(\phi U)\right\|_{L^{2}\left(E_{k_{j}}\right)}
\end{aligned}
$$

where $Y_{j}=E_{k_{j}} \cap \operatorname{supp} \phi$.

Then by comparing with (2.13)

$$
\left(M^{n}\left|E_{k_{j}}\right|\right)^{-\theta} \leq C_{\theta}\left(\|W\|_{L^{n}\left(Y_{j}\right)}+\left|E_{k_{j}}\right|^{\frac{\varepsilon}{n^{(n+\varepsilon)}}}\left\||x|^{-1}\right\|_{L^{n+\varepsilon}\left(Y_{j}\right)}\right)
$$

where $C_{\theta}$ is a new positive constant depending only on $n$ and $\theta$.

Since $M>1$ and $M^{n}\left|E_{k_{j}}\right| \geq 1$, we get

$$
\left(M^{n}\left|E_{k_{j}}\right|\right)^{-\theta-\frac{\varepsilon}{n(n+\varepsilon)}} \leq C_{\theta}\left(\|W\|_{L^{n}\left(Y_{j}\right)}+\left\||x|^{-1}\right\|_{L^{n+\varepsilon}\left(Y_{j}\right)}\right) .
$$

We recall that $\theta$ is any real number such that $\theta>\frac{n-2}{n(n-1)}$. In paricular, for

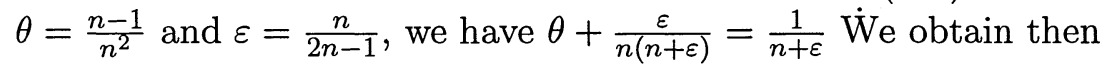

$$
\left(M^{n}\left|E_{k_{j}}\right|\right)^{-\frac{1}{n+\varepsilon}} \leq C\left(\|W\|_{L^{n}\left(Y_{j}\right)}+\left\||x|^{-1}\right\|_{L^{n+\varepsilon}\left(Y_{j}\right)}\right)
$$

that's

$$
\left(M^{n}\left|E_{k_{j}}\right|\right)^{-1} \leq C\left(\|W\|_{L^{n}\left(Y_{j}\right)}^{n+\varepsilon}+\left\||x|^{-1}\right\|_{L^{n+\varepsilon}\left(Y_{j}\right)}^{n+\varepsilon}\right)
$$

where $C$ is a positive constant depending only on $n$.

Since $Y_{j}$ are pairwise disjoint, by taking the sum over $j$ and using (2.12), we get, with a new positive constant $C$ depending only on $n$,

$$
\|W\|_{L^{n}(\operatorname{supp} \phi)}^{n+\varepsilon}+\left\||x|^{-1}\right\|_{L^{n+\varepsilon}(\operatorname{supp} \phi)}^{n+\varepsilon} \geq C
$$

which is is a contradiction since $\|W\|_{L^{n}(\operatorname{supp} \phi)}+\left\||x|^{-1}\right\|_{L^{n+\varepsilon(\operatorname{supp} \phi)}} \rightarrow 0$ when $R_{0} \rightarrow \infty$. The proof of theorem 1.3 is then complete.

To prove theorem 1.2 we need the following estimate 
Lemma 2.3. Let $p=\frac{14 n-4}{7 n+10}, n \geq 3$, and let

$$
\phi(y)=-\log (y)-\log |\log y|, y \in] 0,1[.
$$

Then, for all $\gamma>1$, and for all $u \in C_{0}^{\infty}\left(B\left(0, \frac{1}{4}\right) \backslash\{0\}\right)$, we have the estimate

$$
\begin{aligned}
\left\|(\log |x|)^{-1} e^{\gamma \phi(|x|)} u\right\|_{L^{2}\left(|x|^{-n} d x\right)}+ & \left\|(\log |x|)^{-1} e^{\gamma \phi(|x|)}|x| \nabla u\right\|_{L^{2}\left(|x|^{-n} d x\right)} \leq \\
& C \gamma^{\delta}\left\|(\log |x|) e^{\gamma \phi(|x|)}|x|^{2} \Delta u\right\|_{L^{p}\left(|x|^{-n} d x\right)}
\end{aligned}
$$

where $\delta=\frac{3 n-2}{4 p}-\frac{3 n+2}{8}$, and $C$ is a positive constant depending only on the dimension $n$.

Proof. In order to prove this lemma we follow a method taken from Jerison [3].

Let's introduce polar coordinates in $\mathbb{R}^{n} \backslash\{0\}$ by setting $x=e^{t} \omega, t \in$ $\mathbb{R}, \omega \in S$ where $S$ denotes the unit sphere in $\mathbb{R}^{n}$. In these coordinates the Laplacian takes the form

$$
e^{2 t} \Delta=\partial_{t}^{2}+(n-2) \partial_{t}+\Delta_{\omega}
$$

where $\Delta_{\omega}$ is the Laplace-Beltrami operator in $S$. that

Define the operator $\Lambda$ by $\Lambda=\left(\frac{(n-2)^{2}}{4}-\Delta_{\omega}\right)^{1 / 2}$. It follows from (2.15)

$$
e^{2 t} \Delta=\left(\partial_{t}+\frac{n-2}{2}-\Lambda\right)\left(\partial_{t}+\frac{n-2}{2}+\Lambda\right) .
$$

Let $L=\partial_{t}+\frac{n-2}{2}-\Lambda$, and set $\left.\psi(t)=-\phi\left(e^{t}\right)=t+\log |t|, t \in\right]-\infty,-1[$. We shall prove the following estimate on $L$

$$
\left\|t^{-1} e^{-\gamma \psi(t)} u\right\|_{L^{2}(d t d \omega)} \leq C \gamma^{\delta}\left\|t e^{-\gamma \psi(t)} L u\right\|_{L^{p}(d t d \omega)}
$$

for all $u \in C_{0}^{\infty}(]-\infty,-1[\times S)$. Here $d t$ and $d \omega$ denote the natural measures of $\mathbb{R}$ and $S$ respectively.

As we will see it later, the estimate (2.14) in lemma 2.3 is a direct consequence of (2.17).

Define $L_{\gamma}$ by $L_{\gamma} u=e^{-\gamma \psi(t)} L\left(e^{\gamma \psi(t)} u\right), u \in C_{0}^{\infty}(]-\infty,-1[\times S)$. Then (2.17) is equivalent to

$$
\left\|t^{-1} u\right\|_{L^{2}(d t d \omega)} \leq C \gamma^{\delta}\left\|t L_{\gamma} u\right\|_{L^{p}(d t d \omega)}
$$


Unique continuation for differential equations of Schrödinger's type 311

We recall that the spectrum of $\Delta_{\omega}$ as an operator in $L^{2}(S)$ is $\{-k(k+$ $n-2), k \in \mathbb{N}\}$, and for each eigenvalue the corresponding eigenspace is $E_{k}$, the space of spherical harmonics of degree $k$. It follows that the spectrum of the operator $\left(\frac{n-2}{2}-\Lambda\right)$ is $\{-k, k \in \mathbb{N}\}$ with $E_{k}$ as corresponding eigenspaces. Then, for $v \in C_{0}^{\infty}(S)$,

$$
\left(\frac{n-2}{2}-\Lambda\right) v=-\sum_{k \geq 0} k \pi_{k} v
$$

where $\pi_{k}$ is the projection operator from $L^{2}(S)$ to $E_{k}$.

Then the operator $L_{\gamma}$ takes the form

$$
L_{\gamma}=\sum_{k \geq 0}\left(\partial_{t}+\gamma \psi^{\prime}(t)-k\right) \pi_{k}
$$

We need the following estimates on $\pi_{k}$ due to Sogge [5]:

$$
\left\|\pi_{k} v\right\|_{L^{q}(S)} \leq C k^{1-2 / n}\|v\|_{L^{q^{\prime}}(S)}
$$

where $q=\frac{2 n}{n-2}$, and $1 / q^{\prime}=1-1 / q=\frac{2 n}{n+2}, n \geq 3$.

Let $\left\{a_{k}\right\}$ be a sequence in $\mathbb{R}$ with $\left|a_{k}\right| \leq 1$. We have by Hölder's inequality, for all $M \leq N$,

$$
\left\|\sum_{M}^{N} a_{k} \pi_{k} v\right\|_{L^{2}(S)}^{2} \leq\left(\sum_{M}^{N}\left|a_{k}\right|^{2}\left\|\pi_{k} v\right\|_{L^{q}(S)}\right)\|v\|_{L^{q^{\prime}(S)}},
$$

and by $(2.20)$

$$
\left\|\sum_{M}^{N} a_{k} \pi_{k} v\right\|_{L^{2}(S)} \leq N^{(n-2) / 2 n}\left(\sum_{M}^{N}\left|a_{k}\right|^{2}\right)^{1 / 2}\|v\|_{L^{q^{\prime}}(S)} .
$$

If we interpolate with the trivial estimate $\left\|\sum_{M}^{N} a_{k} \pi_{k} v\right\|_{L^{2}(S)} \leq\|v\|_{L^{2}(S)}$, we get

$$
\left\|\sum_{M}^{N} a_{k} \pi_{k} v\right\|_{L^{2}(S)} \leq\left(N^{(n-2) / 2}\left(\sum_{M}^{N}\left|a_{k}\right|^{2}\right)^{n / 2}\right)^{1 / r-1 / 2}\|v\|_{L^{r}(S)}
$$

for all $r$ such that $\frac{2 n}{n+2} \leq r \leq 2$. 
In particular, if we take $a_{k}=1$ and $a_{j}=0$ if $j \neq k$, we have

$$
\left\|\pi_{k} v\right\|_{L^{2}(S)} \leq C k^{(n-2)(2-r) / 4 r}\|v\|_{L^{r}(S)}
$$

for all $r$ such that $\frac{2 n}{n+2} \leq r \leq 2$.

Let $N=[2 \gamma]+1$, and define the operators $P_{\gamma}^{+}, P_{\gamma}^{-}$by

$$
P_{\gamma}^{+}=\sum_{k>N} \pi_{k} \quad \text { and } \quad P_{\gamma}^{-}=\sum_{k \leq N} \pi_{k}
$$

In order to prove (2.18) we split it in two pieces: first we shall prove

$$
\left\|t^{-1} P_{\gamma}^{+} u\right\|_{L^{2}(d t d \omega)} \leq C\left\|t L_{\gamma} u\right\|_{L^{p}(d t d \omega)}
$$

next

$$
\left\|t^{-1} P_{\gamma}^{-} u\right\|_{L^{2}(d t d \omega)} \leq C \gamma^{\delta}\left\|t L_{\gamma} u\right\|_{L^{p}(d t d \omega)}
$$

Thus it will suffice to take the sum of the two estimates to have (2.18) since $P_{\gamma}^{+} u+P_{\gamma}^{-} u=u$.

Let's now prove $(2.18)^{\prime}$.

By solving a first order ordinary differential equation, we get from (2.19), for all $u \in C_{0}^{\infty}(]-\infty,-1\left[\times S^{n-1}\right)$, and all $k>N$,

$$
\pi_{k} u(t, \omega)=-\int_{-\infty}^{+\infty} H(s-t) e^{k(t-s)+\gamma(\psi(s)-\psi(t))} \pi_{k} L_{\gamma} u(s, \omega) d s
$$

where $H(z)=1$ if $z \geq 0$ and $H(z)=0$ if $z<0$.

But for $k>N$ we have

$$
H(s-t) e^{k(t-s)+\gamma(\psi(s)-\psi(t))} \leq e^{-\frac{1}{2} k|t-s|}
$$

for all $s, t \in]-\infty,-1[$.

Hence by taking the $L^{2}(S)$-norm in $(2.23)$

$$
\left\|\pi_{k} u(t, .)\right\|_{L^{2}(S)} \leq \int_{-\infty}^{+\infty} e^{-\frac{1}{2} k|t-s|}\left\|\pi_{k} L_{\gamma} v(s, .)\right\|_{L^{2}(S)} d s,
$$

and by (2.22) (with $r=p=\frac{14 n-4}{7 n+10}$ )

$$
\left\|\pi_{k} u(t, .)\right\|_{L^{2}(S)} \leq C k^{(n-2)(2-p) / 4 p} \int_{-\infty}^{+\infty} e^{-\frac{1}{2} k|t-s|}\left\|L_{\gamma} u(s, .)\right\|_{L^{p}(S)} d s .
$$


Unique continuation for differential equations of Schrödinger's type 313

Now if we apply Young's inequality we obtain

$$
\left\|\pi_{k} u\right\|_{L^{2}(d t d \omega)} \leq C k^{(n-2)(2-p) / 4 p}\left(\int_{-\infty}^{+\infty} e^{-\frac{a}{2} k|z|} d z\right)^{1 / a}\left\|L_{\gamma} u\right\|_{L^{p}(d t d \omega)}
$$

where $1 / a=3 / 2-1 / p$.

But

$$
\left(\int_{-\infty}^{+\infty} e^{-\frac{a}{2} k|z|} d z\right)^{1 / a} \leq C k^{-3 / 2+1 / p}
$$

hence

$$
\left\|\pi_{k} u\right\|_{L^{2}(d t d \omega)}^{2} \leq C k^{-2+n(1 / p-1 / 2)}\left\|L_{\gamma} u\right\|_{L^{p}(d t d \omega)}^{2} .
$$

It follows by summing over $k>N$,

$$
\sum_{k>N}\left\|\pi_{k} u\right\|_{L^{2}(d t d \omega)}^{2} \leq C\left(\sum_{k>N} k^{-2+n(1 / p-1 / 2)}\right)\left\|L_{\gamma} u\right\|_{L^{p}(d t d \omega)}^{2} .
$$

Since $1 / p=\frac{7 n+10}{14 n-4}$ we have $-2+n(1 / p-1 / 2)<-1$. Then

$$
\sum_{k>N} k^{-2+n(1 / p-1 / 2)} \leq C
$$

so

$$
\left\|P_{\gamma}^{+} u\right\|_{L^{2}(d t d \omega)} \leq C\left\|L_{\gamma} u\right\|_{L^{p}(d t d \omega)}
$$

which is better than $(2.18)^{\prime}$.

Now we shall prove $(2.18)^{\prime \prime}$.

Let $u \in C_{0}^{\infty}(]-\infty,-1[\times S)$. Fix $\left.t \in\right]-\infty,-1\left[\right.$, and set $M=\left[\gamma \psi^{\prime}(t)\right]+1$.

By solving a first order ODE, we have from (2.19), for all $k \in \mathbb{N}$ with $M \leq k<N$,

$$
\pi_{k} u(t, \omega)=-\int_{-\infty}^{+\infty} H(s-t) T_{k}(s, t) \pi_{k} L_{\gamma} u(s, \omega) d s
$$

where $T_{k}(s, t)=e^{k(t-s)+\gamma(\psi(s)-\psi(t))}$ and where $H(z)=1$ if $z \geq 0, H(z)=0$ if $z \leq 0$.

In the same manner, for $k \leq M-1$, one has

$$
\pi_{k} u(t, \omega)=\int_{-\infty}^{+\infty} H(t-s) T_{k}(s, t) \pi_{k} L_{\gamma} u(s, \omega) d s
$$


We recall that $\psi(t)=t+\log |t|$. Then we have by Taylor's formula, for all $s \in]-\infty,-1[$,

$$
\psi(s)-\psi(t)=\psi^{\prime}(t)(s-t)-\frac{1}{2\left|s_{0}\right|^{2}}(s-t)^{2}
$$

where $s_{0}$ is a real number between $s$ and $t$.

It follows that

$$
\gamma(\psi(s)-\psi(t)) \leq M|s-t|-\frac{\gamma}{2|t|^{2}}(s-t)^{2} \quad \text { if } \quad s>t
$$

and

$$
\gamma(\psi(s)-\psi(t)) \leq-(M-1)|s-t|-\frac{\gamma}{2|s|^{2}}(s-t)^{2} \text { if } s<t .
$$

Hence for $k \geq M$ we have

$$
H(s-t) T_{k}(s, t) \leq e^{-\frac{\gamma}{2|t|^{2}}(s-t)^{2}} \cdot e^{-|k-M||s-t|}
$$

for all $s \in]-\infty,-1[$.

And if $k \leq M-1$ we have

$$
H(t-s) T_{k}(s, t) \leq e^{-\frac{\gamma}{2|s|^{2}}(s-t)^{2}} \cdot e^{-|M-k-1||s-t|}
$$

for all $s \in]-\infty,-1[$.

By taking the sum over $k(M \leq k \leq N)$ in (2.24) and passing to the $L^{2}(S)$-norm we obtain

$$
\left\|\sum_{k=M}^{N} \pi_{k} u(t, .)\right\|_{L^{2}(S)} \leq \int_{-\infty}^{+\infty}\left\|\sum_{k=M}^{N} H(s-t) T_{k}(s, t) \pi_{k} L_{\gamma} u(s, .)\right\|_{L^{2}(S)} d s
$$

We have from (2.21) with $r=p=\frac{14 n-4}{7 n+10}$ and $a_{k}=H(s-t) T_{k}(s, t)$ (note here that $\left|a_{k}\right| \leq 1$ by (2.26)),

$$
\begin{aligned}
& \left\|\sum_{k=M}^{N} H(s-t) T_{k}(s, t) \pi_{k} L_{\gamma} u(s, .)\right\|_{L^{2}(S)} \leq \\
& \quad C\left(\gamma^{(n-2) / 2}\left(\sum_{k=M}^{N} H(s-t)\left|T_{k}(s, t)\right|^{2}\right)^{n / 2}\right)^{1 / p-1 / 2}\left\|L_{\gamma} u(s, .)\right\|_{L^{p}(S)} .
\end{aligned}
$$


Unique continuation for differential equations of Schrödinger's type 315

But from (2.26) we have

$$
\sum_{k=M}^{N} H(s-t)\left|T_{k}(s, t)\right|^{2} \leq C|s-t|^{-1} e^{-\frac{\gamma}{|t|^{2}}(s-t)^{2}} .
$$

Then

$$
\begin{aligned}
& \| \sum_{k=M}^{N} H(s-t) T_{k}(s, t) \pi_{k} L_{\gamma} u(s, .) \\
& \leq \\
& C \nu^{2}(S) \\
& C \gamma^{\alpha}|s-t|^{-\beta} e^{-\frac{\beta \gamma}{|t|^{2}}(s-t)^{2}}\left\|L_{\gamma} u(s, .)\right\|_{L^{p}(S)}
\end{aligned}
$$

where $\alpha=\frac{(n-2)(2-p)}{4 p}$ and $\beta=\frac{n(2-p)}{4 p}$.

We have

$$
e^{-\frac{\beta \gamma}{|t|^{2}}(s-t)^{2}} \leq C_{j}\left(1+\frac{\beta \gamma}{|t|^{2}}(s-t)^{2}\right)^{-j} \text { for all } j>0 .
$$

In particular, for $j=1 / 2$,

$$
e^{-\frac{\beta \gamma}{|t|^{2}}(s-t)^{2}} \leq C|t|(1+\sqrt{\gamma}|s-t|)^{-1} .
$$

Therefore

$$
\left\|\sum_{k=M}^{N} H(s-t) T_{k}(s, t) \pi_{k} L_{\gamma} u(s, .)\right\|_{L^{2}(S)} \leq \frac{C \gamma^{\alpha}|t||| L_{\gamma} u(s, .) \|_{L^{p}(S)}}{(1+\sqrt{\gamma}|s-t|)|s-t|^{\beta}},
$$

and by $(2.28)$

$$
|t|^{-1}\left\|\sum_{k=M}^{2 \gamma} \pi_{k} u(t, .)\right\|_{L^{2}(S)} \leq C \int_{-\infty}^{+\infty} \frac{\gamma^{\alpha}\left\|L_{\gamma} u(s, .)\right\|_{L^{p}(S)}}{(1+\sqrt{\gamma}|s-t|)|s-t|^{\beta}} d s .
$$

Now it remains to estimate the part $\sum_{k=0}^{M-1} \pi_{k} u(t,$.$) . If we start from (2.25)$ instead of (2.24) and follow the same steps as in the proof of (2.29) and using (2.27) instead of (2.26), we get without difficulty:

$$
\left\|\sum_{k=0}^{M-1} \pi_{k} u(t, .)\right\|_{L^{2}(S)} \leq C \int_{-\infty}^{+\infty} \frac{\gamma^{\alpha}|s|\left\|L_{\gamma} u(s, .)\right\|_{L^{p}(S)}}{(1+\sqrt{\gamma}|s-t|)|s-t|^{\beta}} d s
$$


and by adding to (2.29),

$$
|t|^{-1}\left\|P_{\gamma}^{-} u(t, .)\right\|_{L^{2}(S)} \leq C \int_{-\infty}^{+\infty} \frac{\gamma^{\alpha}\left\|s L_{\gamma} u(s, .)\right\|_{L^{p}(S)}}{(1+\sqrt{\gamma}|s-t|)|s-t|^{\beta}} d s
$$

where $P_{\gamma}^{-}=\sum_{k=0}^{N} \pi_{k}$.

Now if we apply Young's inequality we obtain

$$
\left\|t^{-1} P_{\gamma}^{-} u\right\|_{L^{2}(d t d \omega)} \leq C \gamma^{\alpha}\left(\int_{-\infty}^{+\infty} \frac{d z}{(1+\sqrt{\gamma}|z|)^{a}|z|^{\beta a}}\right)^{1 / a}\left\|t L_{\gamma} u\right\|_{L^{p}(d t d \omega)}
$$

where $1 / a=3 / 2-1 / p$.

But

$$
\left(\int_{-\infty}^{+\infty} \frac{d z}{(1+\sqrt{\gamma}|z|)^{a}|z|^{\beta a}}\right)^{1 / a} \leq C \gamma^{-\frac{1}{2 a}+\frac{\beta}{2}}
$$

hence

$$
\left\|t^{-1} P_{\gamma}^{-} u\right\|_{L^{2}(d t d \omega)} \leq C \gamma^{-\frac{1}{2 a}+\frac{\beta}{2}+\alpha}\left\|t L_{\gamma} u\right\|_{L^{p}(d t d \omega)} .
$$

This achieves the proof of $(2.18)^{\prime \prime}$ since $-\frac{1}{2 a}+\frac{\beta}{2}+\alpha=\frac{3 n-2}{4 p}-\frac{3 n+2}{8}=\delta$. The proof of (2.18) is then complete, and (2.17) follows since it is equivalent to (2.18).

Now we will conclude the estimate (2.14) in lemma 2.2 from (2.17). In polar coordinates, (2.14) can be written

$$
\begin{array}{r}
\left\|t^{-1} e^{-\gamma \psi(t)} u\right\|_{L^{2}(d t d \omega)}+\left\|t^{-1} e^{t-\gamma \psi(t)} \nabla u\right\|_{L^{2}(d t d \omega)} \leq \\
C \gamma^{\delta}\left\|t e^{2 t-\gamma \psi(t)} \Delta u\right\|_{L^{p}(d t d \omega)} .
\end{array}
$$

We have by $(2.16)$

$$
e^{2 t} \Delta=L\left(\partial_{t}+\Lambda+\frac{n-2}{2}\right) .
$$

Set $T=\partial_{t}+\Lambda+\frac{n-2}{2}$, and let $u \in C_{0}^{\infty}(]-\infty,-1[\times S)$. If we apply (2.17) to the function $T u$ instead of $u$, we get

$$
\left\|t^{-1} e^{-\gamma \psi(t)} T u\right\|_{L^{2}(d t d \omega)} \leq C \gamma^{\delta}\left\|t e^{-\gamma \psi(t)} L T u\right\|_{L^{p}(d t d \omega)}
$$

that's

$$
\left\|t^{-1} e^{-\gamma \psi(t)} T u\right\|_{L^{2}(d t d \omega)} \leq C \gamma^{\delta}\left\|t e^{2 t-\gamma \psi(t)} \Delta u\right\|_{L^{p}(d t d \omega)}
$$


Unique continuation for differential equations of Schrödinger's type 317

Now it remains to check that

$$
\begin{aligned}
&\left\|t^{-1} e^{-\gamma \psi(t)} u\right\|_{L^{2}(d t d \omega)}+\left\|t^{-1} e^{t-\gamma \psi(t)} \nabla u\right\|_{L^{2}(d t d \omega)} \leq \\
& C\left\|t^{-1} e^{-\gamma \psi(t)} T u\right\|_{L^{2}(d t d \omega)} .
\end{aligned}
$$

An easy computation shows that

$$
\begin{aligned}
e^{2 t}\|\nabla u(t, .)\|_{L^{2}(S)}^{2}=\left\|\partial_{t} u(t, .)\right\|_{L^{2}(S)}^{2} & +\left\|\left(-\Delta_{\omega}\right)^{1 / 2} u(t, .)\right\|_{L^{2}(S)}^{2} \\
& \leq\left\|\partial_{t} u(t, .)\right\|_{L^{2}(S)}^{2}+\|\Lambda u(t, .)\|_{L^{2}(S)}^{2},
\end{aligned}
$$

hence if we multiply both sides by $t^{-2} e^{-2 \gamma \psi(t)}$ and integrate with respect to $t$ we get

$$
\begin{aligned}
&\left\|t^{-1} e^{t-\gamma \psi(t)} \nabla u\right\|_{L^{2}(d t d \omega)}^{2} \leq \\
&\left\|t^{-1} e^{-\gamma \psi(t)} \partial_{t} u\right\|_{L^{2}(d t d \omega)}^{2}+\left\|t^{-1} e^{-\gamma \psi(t)} \Lambda u\right\|_{L^{2}(d t d \omega)}^{2}
\end{aligned}
$$

On the other hand, an elementary integration by parts gives

$$
\begin{gathered}
\left\|t^{-1} e^{-\gamma \psi(t)} T u\right\|_{L^{2}(d t d \omega)}^{2} \geq \\
\left\|t^{-1} e^{-\gamma \psi(t)} \partial_{t} u\right\|_{L^{2}(d t d \omega)}^{2}+\left\|t^{-1} e^{-\gamma \psi(t)} \Lambda u\right\|_{L^{2}(d t d \omega)}^{2}+ \\
\frac{(n-2)^{2}}{4}\left\|t^{-1} e^{-\gamma \psi(t)} u\right\|_{L^{2}(d t d \omega)}^{2} .
\end{gathered}
$$

Then we obtain

$$
\begin{aligned}
&\left\|t^{-1} e^{-\gamma \psi(t)} u\right\|_{L^{2}(d t d \omega)}+\left\|t^{-1} e^{t-\gamma \psi(t)} \nabla u\right\|_{L^{2}(d t d \omega)} \leq \\
& C\left\|t^{-1} e^{-\gamma \psi(t)} T u\right\|_{L^{2}(d t d \omega)} .
\end{aligned}
$$

Thus (2.30) follows from this estimate and (2.31). The proof of lemma 2.3 is then complete.

Remark 2.4. One can easily see from the proof of Lemma 2.3 , that the estimate (2.14) remains valid for any $p$ such that $\frac{2 n}{n+2} \leq p \leq 2$, with the same dependence of $\delta$ on $p$, i.e., $\delta=\frac{3 n-2}{4 p}-\frac{3 n+2}{8}$. Note here that $\delta=0$ if $p=\frac{6 n-4}{3 n+2}$, this is similar to the dual version of a Carleman-estimate obtained by Jerison [3] (see also Kim [4]). In Lemma 2.3, we have chosen $p=\frac{14 n-4}{7 n+10}$ because this is the smallest exponent allowing us to prove exponential decay of the form (1.3) on the solutions of (1.1) ( $p$ should be chosen such that 
(2.37) in the proof of theorem 1.2 holds ). The choice $p=\frac{2 n}{n+2}+\varepsilon$ gives only a decay of the form

$$
\int_{\left|x-x_{0}\right|<R}\left|D^{\alpha} u(x)\right|^{2} d x=\mathcal{O}\left(e^{-R^{-a}}\right) \text { as } R \rightarrow 0
$$

for some positive $a($ not necessary $\geq 1)$.

Proof of theorem 1.2. Let $u$ as in theorem 1.2. We may suppose that $x_{0}=0$. By hypothesis $u$ is a $W_{l o c}^{2,2}$ function satisfying

$$
\int_{|x|<R}|u(x)|^{2} d x=\mathcal{O}\left(R^{N}\right) \text { for all } N>0 \text { as } R \rightarrow 0 .
$$

Let's recall the following elementary inequality

$$
\int|\nabla v(x)|^{2} d x \leq\left(\int|v(x)|^{2} d x\right)^{1 / 2}\left(\int|\Delta v(x)|^{2} d x\right)^{1 / 2}
$$

which is valid for all $v \in W_{l o c}^{2,2}$ with compact support.

By using a smooth cut-off function and the last inequality, one easily checks that

$$
\int_{|x|<R}|\nabla u|^{2} d x \leq C R^{-2}\|u\|_{W^{2,2}}\left(\int_{|x|<2 R}|u|^{2} d x\right)^{1 / 2}
$$

where $\|u\|_{W^{2,2}}$ is the $W^{2,2}$-norm of $u$ in the ball $B(0,2 R)$.

Hence it follows from (2.32),

$$
\int_{|x|<R}|\nabla u(x)|^{2} d x=\mathcal{O}\left(R^{N}\right) \text { for all } N>0 \text { as } R \rightarrow 0 .
$$

Fix $R>0$ (small enough) and let $\gamma=R^{-1-\varepsilon}$, where $\varepsilon$ is a small positive number to be chosen later. Let $\chi \in C_{0}^{\infty}\left(\mathbb{R}^{n}\right)$ such that $\chi(x)=1$ if $|x|<R$ and $\chi(x)=0$ if $|x|>2 R$. Thus $\chi$ satisfies also $\left|D^{\alpha} \chi(x)\right| \leq C R^{-|\alpha|}$.

The estimate (2.14) in lemma 2.3 was stated for functions in

$$
C_{0}^{\infty}\left(B\left(0, \frac{1}{4}\right) \backslash\{0\}\right)
$$


Unique continuation for differential equations of Schrödinger's type 319

but a standard limiting argument using (2.32), (2.33) and the inequality $|\Delta u(x)| \leq V(x)|\nabla u(x)|$, shows that it is also true for the function $\chi u$. Then

$$
\begin{gathered}
\left\|(\log |x|)^{-1} e^{\gamma \phi(|x|)} \chi u\right\|_{L^{2}\left(|x|^{-n} d x\right)}+ \\
\left\|(\log |x|)^{-1}|x| e^{\gamma \phi(|x|)} \nabla(\chi u)\right\|_{L^{2}\left(|x|^{-n} d x\right)} \leq \\
C \gamma^{\delta}\left\|(\log |x|)|x|^{2} e^{\gamma \phi(|x|)} \Delta(\chi u)\right\|_{L^{p}\left(|x|^{-n} d x\right)}
\end{gathered}
$$

where $\delta=\frac{3 n-2}{4 p}-\frac{3 n+2}{8}$, and $p=\frac{14 n-4}{7 n+10}$.

Since $\chi=0$ when $|x|>2 R=2 \gamma^{-\frac{1}{1+\varepsilon}}$ we get

$$
\begin{gathered}
\left\|(\log |x|)^{-1} e^{\gamma \phi(|x|)} \chi u\right\|_{L^{2}\left(|x|^{-n} d x\right)}+ \\
\left\|(\log |x|)^{-1}|x| e^{\gamma \phi(|x|)} \nabla(\chi u)\right\|_{L^{2}\left(|x|^{-n} d x\right)} \leq \\
C\left\|(\log |x|)|x|^{2-\delta-\varepsilon \delta} e^{\gamma \phi(|x|)} \Delta(\chi u)\right\|_{L^{p}\left(|x|^{-n} d x\right)}
\end{gathered}
$$

which gives

$$
\begin{aligned}
& \left(\int_{|x|<R}\left((\log |x|)^{-1} e^{\gamma \phi(|x|)}|u|\right)^{2}|x|^{-n} d x\right)^{1 / 2}+ \\
& \left(\int_{|x|<R}\left((\log |x|)^{-1}|x| e^{\gamma \phi(|x|)}|\nabla u|\right)^{2}|x|^{-n} d x\right)^{1 / 2} \leq \\
& C\left\|(\log |x|)|x|^{2-\delta-\varepsilon \delta} e^{\gamma \phi(|x|)} \Delta(\chi u)\right\|_{L^{p}\left(|x|^{-n} d x\right)}
\end{aligned}
$$

since $\chi=1$ when $|x|<R$.

We have from the inequality $|\Delta u(x)| \leq V(x)|\nabla u(x)|$,

$$
\begin{aligned}
& \left\|(\log |x|)|x|^{2-\delta-\varepsilon \delta} e^{\gamma \phi(|x|)} \Delta(\chi u)\right\|_{L^{p}\left(|x|^{-n} d x\right)} \leq \\
& \left(\left.\left.\int_{|x|<R}|(\log |x|)| x\right|^{2-\delta-\varepsilon \delta} e^{\gamma \phi(|x|)} V(x)|\nabla u(x)|\right|^{p}|x|^{-n} d x\right)^{1 / p}+ \\
& \left(\left.\left.\int_{|x|>R}|(\log |x|)| x\right|^{2-\delta-\varepsilon \delta} e^{\gamma \phi(|x|)} \Delta(\chi u)\right|^{p}|x|^{-n} d x\right)^{1 / p}
\end{aligned}
$$


Let $q=\frac{7 n-2}{6}$, and note here that $\frac{1}{p}=\frac{1}{q}+\frac{1}{2}$. By Hölder's inequality we have

$$
\begin{aligned}
&\left(\left.\left.\int_{|x|<R}|(\log |x|)| x\right|^{2-\delta-\varepsilon \delta} e^{\gamma \phi(|x|)} V(x)|\nabla u(x)|\right|^{p}|x|^{-n} d x\right)^{1 / p} \leq \\
&\left(\int_{|x|<R}\left((\log |x|)^{2}|x|^{1-\delta-\varepsilon \delta} V\right)^{q}|x|^{-n} d x\right)^{1 / q} \times \\
&\left(\int_{|x|<R}\left((\log |x|)^{-1}|x| e^{\gamma \phi(|x|)}|\nabla u|\right)^{2}|x|^{-n} d x\right)^{1 / 2}
\end{aligned}
$$

But we have

$$
(1-\delta) q=n
$$

Hence

$$
\left(\int_{|x|<R}\left((\log |x|)^{2}|x|^{1-\delta-\varepsilon \delta} V\right)^{q}|x|^{-n} d x\right)^{1 / q}=\left\|(\log |x|)^{2}|x|^{-\varepsilon \delta} V\right\|_{L^{q}(B(0, R))}
$$

where

$$
\left\|(\log |x|)^{2}|x|^{-\varepsilon \delta} V\right\|_{L^{q}(B(0, R))}
$$

is the $L^{q}$-norm of the function $(\log |x|)^{2}|x|^{-\varepsilon \delta} V$ in the ball $B(0, R)$.

We have by hypothesis $V \in L_{l o c}^{r}$ for $r>q$. Then if $\varepsilon$ is sufficiently small, we have $(\log |x|)^{2}|x|^{-\varepsilon \delta} V \in L_{l o c}^{q}$, so

$$
\left\|(\log |x|)^{2}|x|^{-\varepsilon \delta} V\right\|_{L^{q}(B(0, R))} \rightarrow 0 \quad \text { when } R \rightarrow 0 .
$$

In particular, for $R$ sufficiently small

$$
\left\|(\log |x|)^{2}|x|^{-\varepsilon \delta} V\right\|_{L^{q}(B(0, R))} \leq \frac{1}{2 C}
$$

where $C$ is as in (2.34).

Then it follows from (2.36) that

$$
\begin{gathered}
\left(\left.\left.\int_{|x|<R}|(\log |x|)| x\right|^{2-\delta-\varepsilon \delta} e^{\gamma \phi(|x|)} V(x)|\nabla u(x)|\right|^{p}|x|^{-n} d x\right)^{1 / p} \leq \\
\frac{1}{2 C}\left(\int_{|x|<R}\left((\log |x|)^{-1}|x| e^{\gamma \phi(|x|)}|\nabla u|\right)^{2}|x|^{-n} d x\right)^{1 / 2}
\end{gathered}
$$


Now if we combine (2.34) with (2.35) and (2.38), we get

$$
\begin{aligned}
& \left(\int_{|x|<R}\left((\log |x|)^{-1} e^{\gamma \phi(|x|)}|u|\right)^{2}|x|^{-n} d x\right)^{1 / 2}+ \\
& \left(\int_{|x|<R}\left((\log |x|)^{-1}|x| e^{\gamma \phi(|x|)}|\nabla u|\right)^{2}|x|^{-n} d x\right)^{1 / 2} \leq \\
& C\left(\left.\left.\int_{|x|>R}|(\log |x|)| x\right|^{2-\delta-\varepsilon \delta} e^{\gamma \phi(|x|)} \Delta(\chi u)\right|^{p}|x|^{-n} d x\right)^{1 / p}
\end{aligned}
$$

where $C$ is a new positive constant depending only on $n$.

Since $\chi$ is supported in $B(0,2 R)$, and since $\phi$ is a decreasing function, we have

$$
\begin{aligned}
&\left(\left.\int_{|x|>R}|(\log |x|)| x\right|^{2-\delta-\varepsilon \delta} e^{\gamma \phi(x)}|\Delta(\chi u)|^{p}|x|^{-n} d x\right)^{1 / p} \\
& C|\log R| R^{-\frac{n}{p}-\delta-\varepsilon \delta} e^{\gamma \phi(R)}\|u\|_{W^{2, p}}
\end{aligned}
$$

where $\|u\|_{W^{2, p}}$ is the $W^{2, p}$-norm of $u$ in the ball $B(0,2 R)$.

If $R$ is sufficiently small we have $\|u\|_{W^{2, p}}<1$. Then

$$
\begin{aligned}
\left(\left.\left.\int_{|x|>R}|(\log |x|)| x\right|^{2-\delta-\varepsilon \delta} e^{\gamma \phi(|x|)} \Delta(\chi u)\right|^{p}|x|^{-n} d x\right)^{1 / p} \leq \\
C|\log R| R^{-\frac{n}{p}-\delta-\varepsilon \delta} e^{\gamma \phi(R)}
\end{aligned}
$$

and if we compare with (2.39), we get

$$
\begin{aligned}
& \left(\int_{|x|<R}\left((\log |x|)^{-1} e^{\gamma \phi(|x|)}|u|\right)^{2}|x|^{-n} d x\right)^{1 / 2}+ \\
& \left(\int_{|x|<R}\left((\log |x|)^{-1}|x| e^{\gamma \phi(|x|)}|\nabla u|\right)^{2}|x|^{-n} d x\right)^{1 / 2} \leq
\end{aligned}
$$

$C|\log R| R^{-\frac{n}{p}-\delta-\varepsilon \delta} e^{\gamma \phi(R)}$.

On the other hand we have

$$
\begin{aligned}
& \left(\int_{|x|<R}\left((\log |x|)^{-1} e^{\gamma \phi(|x|)}|u|\right)^{2}|x|^{-n} d x\right)^{1 / 2}+ \\
& \left(\int_{|x|<R}\left((\log |x|)^{-1}|x| e^{\gamma \phi(|x|)}|\nabla u|\right)^{2}|x|^{-n} d x\right)^{1 / 2} \geq
\end{aligned}
$$




$$
\begin{aligned}
& e^{\gamma \phi(R / 4)}\left(\left.\int_{|x|<R / 4}|u|^{2}|\log | x\right|^{-2}|x|^{-n} d x\right)^{1 / 2}+ \\
& e^{\gamma \phi(R / 4)}\left(\left.\int_{|x|<R / 4}|\nabla u|^{2}|\log | x\right|^{-2}|x|^{2-n} d x\right)^{1 / 2},
\end{aligned}
$$

then

$$
\begin{aligned}
& \left(\left.\int_{|x|<R / 4}|u|^{2}|\log | x\right|^{-2}|x|^{-n} d x\right)^{1 / 2}+ \\
& \left(\left.\int_{|x|<R / 4}|\nabla u|^{2}|\log | x\right|^{-2}|x|^{2-n} d x\right)^{1 / 2} \leq \\
& C|\log R| R^{-\frac{n}{p}-\delta-\varepsilon \delta} e^{\gamma(\phi(R)-\phi(R / 4))},
\end{aligned}
$$

but if $R$ is small enough we have $\phi(R)-\phi(R / 4) \leq-1$. Hence

$$
\begin{aligned}
& \left(\int_{|x|<R / 4}|u|^{2}|\log | x||^{-2}|x|^{-n} d x\right)^{1 / 2}+ \\
& \left(\left.\int_{|x|<R / 4}|\nabla u|^{2}|\log | x\right|^{-2}|x|^{2-n} d x\right)^{1 / 2} \leq
\end{aligned}
$$$$
C|\log R| R^{-\frac{n}{p}-\delta-\varepsilon \delta} e^{-\gamma}
$$

which is better than the desired result since $\gamma=R^{-1-\varepsilon}$.

Proof of theorem 1.1. This is an immediate consequence of theorem 1.2 and theorem 1.3.

\section{References.}

[1] B. Barcelo, C.E. Kenig, A. Ruiz and C.D. Sogge, Weighted Sobolev inequalities and unique continuation for the Laplacian plus lower order terms, Ill. J. Math. 32 (1988), 230-245.

[2] L. Hörmander, Uniqueness theorems for second order elliptic differential equations, Comm. Partial Differential Equations. 8 (1983), 21-64. 
Unique continuation for differential equations of Schrödinger's type 323

[3] D. Jerison, Carleman inequalities for the Dirac and Laplace operators and unique continuation, Adv. Math. 63 (1986), 118-134.

[4] Y.M. Kim, Carleman inequalities for the Dirac operator and strong unique continuation, Proc. Amer. Math. Soc. 123 (1995), 2103-2112.

[5] C.D. Sogge, Oscillatory integrals and spherical harmonics, Duke Math. J. 53 (1986), 43-65.

[6] T.H. Wolff, Unique continuation for $|\Delta u| \leq V|\nabla u|$ and related problems, Revista Math. Iberoamericana, 6 (1990), 155-200.

[7] T.H. Wolff, A property of measures in $\mathbb{R}^{n}$ and an application to unique continuation, Geom. Funct. Analy. 2 (1992), 225-284.

[8] T.H. Wolff, A counterexample in a unique continuation problem, Comm. Analy. Geom. 2 (1994), 79-102.

Received February 3, 1997.

FACUlté DES SCIENCES

Université DE BRETAGNe OCCIDENTALe,

6, Avenue Le Gorgeu, BP 809

29285 BREST - FRANCE 